\title{
$2 D$ modelling of atmospheric dispersion in the near field at the site of La Hague
}

\author{
H. Berestycki, F. Hamel, N. Dellero', P. Devin ${ }^{1}$ and M. Rennesson ${ }^{1}$ \\ Université Paris VI, Laboratoire d'Analyse Numérique, 187 rue du Chevaleret, \\ 75013 Paris, France \\ ' COGEMA, 2 rue Paul Dautier, 78140 Vélizy-Villacoublay, France
}

\begin{abstract}
To refine assessment of radionuclides concentrations in the environment in the very near field at the La Hague site, COGEMA has decided to develop a dedicated 2D dispersion model. This simplified model aims to provide accurate assessment for the main meteorological situations while assuring a computer answer in a limited time (objective of 20 to 30 minutes on a basic computer for a fine mesh). The model has been developed by the University of Paris VI (Numerical Analysis Laboratory) which has provided the software. In order to test and calibrate this research tool, a comparison progran with the 3D MERCURE software on academic cases bas been launched. Together, a comparison between field measurements of krypton 85, picked up at four monitoring points around the site, and model outputs, has been planned. At the same time, the creation of a user-friendly interface is to be performed to enrich the software, and to facilitate sets of runs for various assumptions (involving model complexity, mesh refinement, meteorological episodes complexity) according to the needs.
\end{abstract}

\section{MOTIVATION AND MODEL ORIENTATION}

Numerous models have been worked out to assess pollutant dispersion in the environment. The first models were based on the analytical resolution of a Gaussian dispersion equation, (from a few kilometres to about 20 kilometres). These simplified models are well adapted to flat terrains (Doury, Pasquill for examples).

More accurate and complex 3D numerical models have been recently developed to fit better with pollutant concentrations in complex canyon situations. These 3D numerical models have also been developed for regional climate simulation including wind field calculations in different meteorological situations. These codes are usually time consuming, depending on the refinement of the mesh and of the meteorological conditions.

By 1999, in the aim of improving the radionuclides concentration assessment in the near field of the La Hague reprocessing site, COGEMA initiated a cooperation with the University of Paris VI, Numerical Analysis Laboratory, to start developing a new model. Considering existing models, the goal was to find an intermediary between Gaussian models and 3D codes, i.e. a compromise between accuracy, speed, robustness and simplicity of use.

To gain in computation time, it has appeared acceptable to select a 2D model, given the site characteristics. The dispersion is evaluated in a vertical plane by the solver of the software, supplemented with a Gaussian lateral loss of material, in order to have finally a real transcription of a 3D dispersion. Landscape and buildings are modelled, in order to take into account turbulence due to ground roughness and eddies behind buildings. Moreover, thermal effects are included, through Boussinesq hypothesis (buoyancy of the atmosphere). All these modules can be activated separately.

The objective would be to provide results in 20 to 30 minutes on a standard $\mathrm{PC}$, in order to facilitate sets of runs for various assumptions.

This 2D model software, called HAG2D, is presently under checking and has been submitted to a qualification program. This paper will present the latest results of the launched program which includes comparison of HAG2D with the validated MERCURE 3D model on one hand, and krypton 85 field measurements on the other hand. Comparisons imply several meteorological conditions. 


\section{MATHEMATICAL MODELLING}

With a view to construct a model which could take into account the needs which have been described above (viz. an accurate near field evaluation, taking into account buildings, topography of the landscape and the need for a user friendly software), we were led to devise several approximations.

The mathematical description consists in two main parts:

- First, a fuid mechanics module aimed at computing the air circulation on the site.

- Second, a transport module designed to compute the atmospheric dispersion of the products on the region that has been selected.

The latter module uses the results of the first one as input data. Both of these modules may incorporate a large variety of phenomena (which will be described below). By far, the main limitation in time and precision is caused by the fluid mechanics module. There, one runs into the classical difficulties and delicate points of scientific computing in this field.

In view of this problem, the main simplification we have imposed is to consider that the average wind flow is two dimensional. We just extrude in the third dimension the landscape of the reference plane section. This therefore also implies a simplification of the landscape. The reference plane in which the computation is carried out is chosen to be that of the dominating wind. Indeed, in view of the dominating atmospheric conditions (and to some extent of the topography) on the La Hague site, this approach seems to be justified.

The landscape in the cross section is defined by topographical data which are used as input. One defines the domain of computation by choosing a limiting vertical height and horizontal extensions. Then, a mesh is automatically generated. The same mesh will be used for both modules of computation. An example of such a mesh, involving about 4700 vertices, is given in figure 2 below. This size of mesh is still somewhat coarse for the required precision.

The next step in the first module is to solve the fluid mechanics equations. There too, there are several options that may be chosen according to requirements of precision and computational time and also depend on the available input data. Some models are designed to give quick estimations and may be used to carry a large number of simulations in a relatively short time, while others are more precise. These can be stored in a library of typical meteorological situations that could be later used to carry rapid and precise computations of dispersion.

In the $2 \mathrm{D}$ approximation, the velocity of the wind is represented by a vector with two components. The basic equations of fluid mechanics are the usual Navier-Stokes equations (mass and momentum conservation). These equations involve the velocity field $\vec{q}$ (in $\mathrm{m}_{\mathrm{s}} \mathrm{s}^{-\mathrm{t}}$ ), the density of the fluid $\rho$ (in $\mathrm{kg} \cdot \mathrm{m}^{-3}$ ), the pressure $p$ (in $\mathrm{kg} \cdot \mathrm{m}^{-1} \cdot \mathrm{s}^{-2}$ ) and the viscosity $v$ (in $\mathrm{m}^{2} \cdot \mathrm{s}^{-1}$ ). One is looking for a stationary solution. The two main issues here are how to take into account the effects of turbulence and the effects of temperature gradients.

Concerning the former, we follow the classical approach to turbulence by determining a stationary mean field $\vec{q}$. There are several possible approximations.

Here, we list these models in order of increasing computational time:

- The simplest method is to take a constant value for the viscosity, called the turbulent viscosity: $v=v_{T}$.

- A second, more refined approximation, is to take the viscosity as a function of the altitude with respect to the ground level. This function is determined by the appropriate meteorological class of atmospheric stability and measurements on the site at various altitudes.

- The third and most complex approximation is to compute the viscosity parameter $v$ as a function of two other quantities : the turbulent kinetic energy $k$ and the dissipation rate of turbulent energy $\varepsilon$. One is thus led to the $k-\varepsilon$ model, a coupled non linear system which involves two more equations for $k$ and $\varepsilon$ [3]. A particular care has to be paid here to the choice of boundary conditions, especially on the ground leve].

In neutral conditions, one does not need to consider the temperature dependence. Then, given the size of the domain, we assume a constant density approximation. The complete model however involves a coupling with temperature. We have chosen a Boussinesq type approximation [1] in which the density $\rho$ is not constant but given by a linear dependence with respect to the temperature $T$ (in $\mathrm{K}$ ) : 


$$
\rho=\rho(T)=\rho_{0}-\frac{\rho_{0}}{T_{0}}\left(T-T_{0}\right),
$$

where $T_{0}$ is a reference ground temperature (in $\mathrm{K}$ ) and $\rho_{0}$ is the ground density (in $\mathrm{kg} \cdot \mathrm{m}^{-3}$ ).

Then the Navier-Stokes equations are coupled with a heat and transport equations which are more conveniently written in terms of the potential temperature (in $\mathrm{K}$ ) given by $\theta \cong T+\left(g / C_{p}\right) . z$, with $g$ the standard gravity $\left(=9.8 \mathrm{~m} \cdot \mathrm{s}^{-2}\right), C_{p}$ the specific heat at constant pressure (in $\mathrm{m}^{2} \cdot \mathrm{s}^{-2} \cdot \mathrm{K}^{-1}$ ), $z$ the height (in $\mathrm{m}$ ).

Next, the second module takes up the task of computing dispersion under the effects of diffusion and transport by the wind obtained in the previous module. This is modelled by a transport-diffusion equation and takes into account a large array of physical phenomena involving many further parameters: loss by radioactive decay ; accumulation of deposit (in case of non rare gas) in wet or dry conditions, loss by migration into soil; atmospheric resuspension of the deposit).

\section{DESCRIPTION OF THE APPLICATION PROGRAM}

For carrying the numerical resolution of the system of partial differential equations, we apply a finite element method. We use the equation solver FreeFem+ software which has been developed at University of Paris VI and at INRIA in France [6]. This carries the finite element method using a conjugate gradient algorithm.

The minimal hardware requirements are a PC with PII type processor, $400 \mathrm{MHz}$ and $128 \mathrm{Mo}$ memory. The software is presently implemented under Linux operating system (e.g. Red Hat 6.0 distribution, F90 compiler, X11 libraries included).

The limiting vertical height, the horizontal extensions as well as the refinement of the mesh are parameters that the user may modify. More precisely, the user has the option to define regions near the boundary where the mesh is refined as well as the overall degree of refinement.

Aside the topographical data, the software requires several data as input files: the profile of the horizontal speed of incident wind on the domain, the temperature profile, the profiles of $k$ and $\varepsilon$ or alternatively the viscosity profile at the entry. Then, the user has the option to choose the turbulence model (constant, given varying viscosity or $k-\varepsilon$ model), and coupling with the thermal model. In all these models, parameters are available to play on the speed/accuracy of the computation.

As for dispersion, the following effects are retained as choice options: sources, flows and length of emissions, vertical deposit due to rain, nature of the product, radioactive decay rate, migration rate into the ground, wet and dry deposit, resuspension..

Several output visualizations are available, either during the computations, or once the results have been obtained. They give both the instantaneous established velocity fields and Atmospheric Transfer Coefficients (ATC). In particular, one can visualize the ATC on the ground as a function of the distance to the source, corresponding to a special meteorological situation.

\section{RESULTS OF THE COMPARISON PROGRAM}

The two-step comparison program is described below. The results are presented as comparative quantitative outputs and qualitative $2 \mathrm{D}$ representations on the terrain.

\subsection{Methodology}

The comparison program has been divided into two consecutive parts. First, the model results are compared with an other validated dispersion model, in order to check the good physical consistency of the outputs.

The choice of the comparison tool fell on the 3D software called MERCURE, developed by EDF, which is mainly dedicated to dispersion over complex terrain, and is part of the SMEDIS validation program. 
The results of both models are then compared to field measurements, collected at four different monitoring stations from around the site. A first set of scenarios is used to adjust parameters for each class of meteorological conditions and a second one to ensure the consistency of this adaptation.

\subsubsection{Validation through comparison with a $3 D$ model}

The tests used are simplified cases, which allow splitting up the model into several modules corresponding to the different physical phenomena being modelled. This benchmark consists of a set of six validation tests, in order to control the modelling of the following modules: Navier-Stokes for the wind field calculation, transport-diffusion of the radionuclides concentrations, thermal phenomena, and turbulence fluctuations.

For this part, MERCURE software is run in 2D mode.

\subsubsection{Validation on real cases}

\subsubsection{Collecting the inputs}

The inputs were identical for both codes, HAG2D and MERCURE, namely input data and $2 \mathrm{D}$ geometric sections. Starting from the topography, a 2D mesh is constructed by automatic generation.

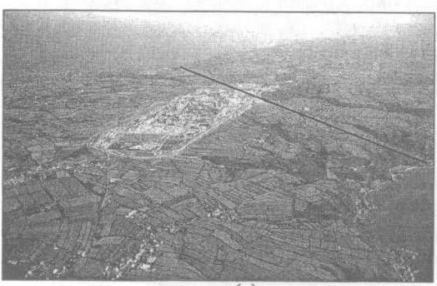

(a)

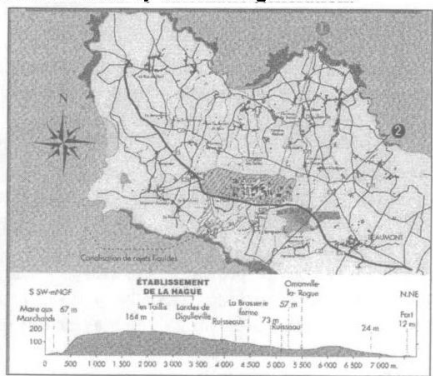

(b)

Figure I: (a) aerial picture of the La Hague site with location of the SSW-NNE cross-section, (b) up: the cross-section (1) is the NNW-SSE cut crossing Herqueville and Digulleville. (b) down: topography details for this cut.

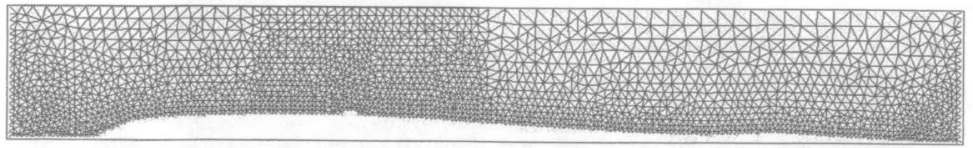

Figure 2: Example of the mesh built from the SSW-NNE (Herqueville-Digulleville) cross-section, about 4700 vertices (2500 cells).

The input data consists of release data and meteorological data, namely vertical profiles of wind, temperature, and eddy viscosity. All the meteorological parameters are collected on site: a combination of a $10 \mathrm{~m}$ mast, sodar, and pluviometer for gathering and storing of wind values (direction and strength) at seven altitudes between 10 and $200 \mathrm{~m}$, temperature gradient, moisture rate and vertical wind speed standard deviation. The data are transferred in real time to the Environment Checkpoint, in order to feed the meteorological pre-processor. Data have to be computed afterwards to complete the input data sets of the code. To overcome missing information, comparison with predefined characteristics of Pasquill 
stability classes may be performed. The main difficulty is to reconstruct the thermal gradient profiles, because few information (only at ground and $10 \mathrm{~m}$ level) are available. Theoretical profiles are then chosen in relation with the stability class.

In order to select the meteorological data for the comparison, an analysis was carried out to retain a set of 27 discharge scenarios over a four month period (March to June 2000), including meteorological conditions stability, durability and concentration level above a fixed threshold [4].

The corresponding profiles were reconstructed from the meteorological data thanks to interpolation laws taken from literature (Laiktman, O'Brien [2][5]), and differentiated for the six atmosphere stability classes of Pasquill. Then, for both models, the approximations were keyed using experimental data.

\subsubsection{Collecting the krypton 85 concentrations}

The outputs were measured krypton 85 concentrations. The four monitoring stations are spread out over the area, from $1.1 \mathrm{~km}$ for the nearest to $5.5 \mathrm{~km}$ for the farthest one. The data, updated every 18 seconds, are transferred directly and continuously to the Environment Checkpoint.

\section{Simulations results}

For the inter-code comparison, the compared output values are wind, temperature, eddy viscosity profiles, concentrations and ATC for different abscissas along the $\mathrm{x}$-axis. This leads to comparative curves, as it is not consistent to estimate the deviation between two points on differently structured grid (structured rectangular for MERCURE and unstructured triangular for HAG2D meshes). For the real scenarios, one must be very careful since the only available values are measurements at ground level (about $5 \mathrm{~m}$ high).

\subsubsection{Academic benchmark}

A good correlation was obtained through the benchmark. The maximum deviation between MERCURE and HAG2D is of magnitude of about $20 \%$, as illustrated with the figures below, in a simple case.
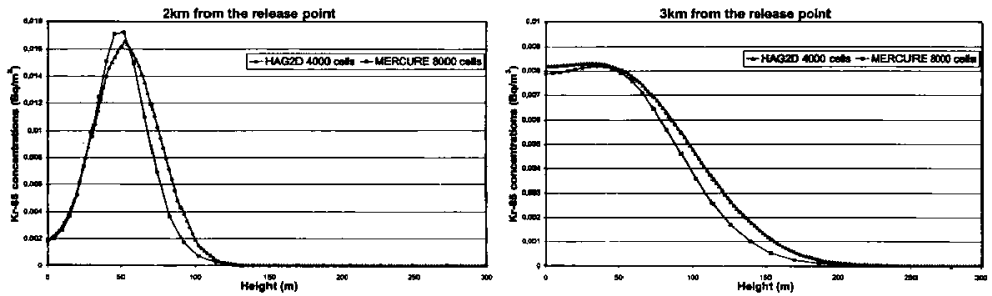

Figure 3: Comparison of MERCURE and HAG2D software outputs on an academic case (dispersion over flat terrain, laminar flow), for two distances from the release point.

This allowed to consider that HAG2D modelling is mathematically and numerically satisfying. Now, the aim is to test the model with respect to real release episodes.

\subsubsection{Simulations of real discharges}

At the moment, two situations have been considered: Digulleville (2.6 km NNE from site, relatively flat terrain, entering SSW wind) and Herqueville (1.1 km SSW from site, in a canyon, entering NNE wind), for neutral (D Pasquill stability class) for neutral and relatively stable conditions (E class). The results are presented below for Digulleville in the neutral case (equilibrium between thermal and mechanical effects). The calculated ATC values far all different stability classes should be weighted by the observed frequencies of such meteorological situations over the year to calculate the average annual Atmospheric Transfert Coefficient. First results concern simple and well known situations. As shown on figure 4 and 
table 1 , these results are encouraging (same order of magnitude, maximum deviation of about $35 \%$ for HAG2D and $20 \%$ for MERCURE).

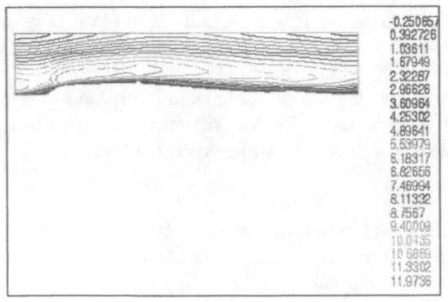

Figure 4: HAG2D results for neutral diffusion conditions on the SSW-NNE cross-section through Herqueville and Digulleville ( $1000 \mathrm{~m}$ high, $7000 \mathrm{~m}$ long), for the complete model: isolines of the horizontal wind component (in $\mathrm{m} \cdot \mathrm{s}^{-1}$ ).

This picture shows the influence of landscape through wind velocities: weak wind before and on the shape, reaching maximum speed (12 $\mathrm{m}, \mathrm{s}^{-1}$ ) at nearby $500 \mathrm{~m}$ above sea-level and then decreasing with height.

Table 1: Comparison between observed and computed ATC values for Pasquill D stability class.

\begin{tabular}{|c|c|c|c|}
\hline $\operatorname{ATC}\left({\left.\mathrm{s} . \mathrm{m}^{-3}\right)}^{-3}\right.$ & Experimental data & HAG2D & MERCURE \\
\hline Digulleville & $1.9 \mathrm{E}-6$ & $2.55 \mathrm{E}-6$ & $2.30 \mathrm{E}-6$ \\
\hline Herqueville & $2.3 E-6$ & $3.2 \mathrm{E}-6$ & $1.8 \mathrm{E}-6$ \\
\hline
\end{tabular}

\section{CONCLUSIONS AND FUTURE PROSPECTS}

First results of the 2D dispersion modelling in the near field of the La Hague site, launched by COGEMA in 1999 are encouraging. But, if the $2 \mathrm{D}$ approximation allows for a higher level of complexity than a Gaussian model on one hand, it introduces restrictions concerning 3D phenomena on the other hand.

The first obtained results have to be consolidated by further additional comparisons in various cases. The in progress qualification and checking program will be extended and cooperation with the research team will go on until clarification of all treated cases.

In the future, the user-friendly interface on PC which is in development will facilitate the computations. Pre-calculated values of wind field or concentrations distribution for modelled meteorological situations could provide also quicker comparisons with instantaneous field measurements.

\section{Acknowledgements}

The authors would like to especially thank P. Bouland and E. Prian (SGN) for their invaluable contribution in the validation part, and for the large time they spent on MERCURE calculations and comparison aspects.

\section{References}

[1] Drazin, W.H. Reid, Hydrodynamic Stability (Cambridge Univ. Press, Cambridge, 1981).

[2] S.R. Hanna, G.A. Briggs, R.P. Hosker Jr, Hanbook on Atmospheric Diffusion (Technical Information Center - U.S. Dpt of Energy, 1966).

[3] B. Mohammadi, O. Pironneau, Analysis of the $k-\varepsilon$ model (Masson, Paris and Wiley, New York, 1994).

[4] SGN, Sélection des données relatives à la dispersion du Krypton autour du site de La Hague (Note interne, 2001).

[5] SGN, Méthodologie de caractérisation des conditions météorologiques - Détermination des profils de température, de vitesse et de turbulence (Note inteme, 2001).

[6] Websites of O. Pironneau: http:/www.ann.jussieu.fr/ pironneau/freefem.htm and of F. Hecht :

http://www-rocg.inria.fr/Frederic.Hecht/FreeFemPlus.htm. 